Alper Atamtürk* Deepak Rajan

\title{
On splittable and unsplittable flow capacitated network design arc-set polyhedra
}

Received: August 8, 2000 / Accepted: October 25, 2001

Published online December 6, 2001 - (C) Springer-Verlag 2001

\begin{abstract}
We study the polyhedra of splittable and unsplittable single arc-set relaxations of multicommodity flow capacitated network design problems. We investigate the optimization problems over these sets and the separation and lifting problems of valid inequalities for them. In particular, we give a linear-time separation algorithm for the residual capacity inequalities [19] and show that the separation problem of $c$-strong inequalities [7] is $\mathcal{N} \mathcal{P}$-hard, but can be solved over the subspace of fractional variables only. We introduce two classes of inequalities for the unsplittable flow problems. We present a summary of computational experiments with a branch-and-cut algorithm for multicommodity flow capacitated network design problems to test the effectiveness of the results presented here empirically.
\end{abstract}

\section{Introduction}

Given a network, a set of origin-destination vertex pairs (commodities) and demand data for the commodities, the multicommodity capacitated network design problem is to install integer multiples of some capacity unit on the arcs of the network and route the flow of commodities so that the sum of capacity installation and flow routing costs is minimized while meeting the demands for the commodities. Installing or leasing fiberoptic cables on a telecommunication network, determining the capacities of production lines or warehouses in a production-distribution system, determining the number of engines to power a set of trains on a railroad network can all be viewed as installing capacities on the arcs of a network and routing the flow of commodities on the network.

In many applications flow of a commodity is restricted to run through a single path along the network. This is the case, for instance, in telecommunication networks running asynchronous transfer mode (ATM) protocol, production-distribution with single sourcing, and express package delivery, see $[4,12]$. These problems are generally formulated using a binary flow variable $x_{k a}$ for each commodity-arc pair $(k, a)$ that takes on a value of 1 if the commodity uses the arc, 0 otherwise and an integer capacity variable $y_{a}$. So for each arc of the network there is a capacity constraint of the form

$$
\sum_{k \in K} d_{k} x_{k a} \leq c_{a 0}+c_{a} y_{a}
$$

where $K$ denotes the set of commodities, $d_{k}$ the demand of commodity $k, c_{a 0}$ existing capacity of the arc and $c_{a}$ the unit capacity to install. If the flow of commodities is

A. Atamtürk, D. Rajan: Department of Industrial Engineering and Operations Research, University of California, Berkeley, CA 94720-1777, USA, e-mail: \{atamturk, deepak\} @ieor.berkeley. edu

\footnotetext{
* Supported, in part, by NSF grants DMI-9908705 and DMI-0070127.
} 
allowed to be split among several paths, then the $0-1$ restriction on the flow variables is dropped.

Strong valid inequalities from simple structured relaxations of optimization problems over more complicated sets can be very useful in solving these problems. See for instance $[8-10,20]$ for successful results with this approach. In this paper, we study the convex hull of solutions to constraints of the form (1). We investigate optimization problems over these polyhedra and the separation and lifting problems of valid inequalities for them. Formally, the sets we consider are defined as

$$
\begin{array}{ll}
\text { Splittable flow arc set: } & \mathcal{F}_{S} \equiv\left\{(x, y) \in \mathcal{D}_{S}: \sum_{i \in N} a_{i} x_{i} \leq a_{0}+y\right\} \\
\text { Unsplittable flow arc set: } & \mathcal{F}_{U} \equiv\left\{(x, y) \in \mathcal{D}_{U}: \sum_{i \in N} a_{i} x_{i} \leq a_{0}+y\right\},
\end{array}
$$

where $N \equiv\{1,2, \ldots, n\}$ is an index set and

$$
\mathcal{D}_{S} \equiv\left\{x \in[0,1]^{n}, y \in \mathbb{Z}\right\} \text { and } \mathcal{D}_{U} \equiv\left\{x \in\{0,1\}^{n}, y \in \mathbb{Z}\right\} .
$$

One arrives at $F_{S}$ or $F_{U}$ by dividing (1) by $c_{a}$. The unsplittable flow arc set $\mathcal{F}_{U}$ is a relaxation of the feasible region of the more familiar 0-1 knapsack problem obtained by introducing a general integer variable $y$ with no bounds. Although the formulation of $\mathcal{F}_{U}$ is quite similar to the formulation of the knapsack set, as shown in Sect. 3, its structure is significantly different from the latter. The splittable flow arc set $\mathcal{F}_{S}$ is the relaxation of $\mathcal{F}_{U}$ obtained by allowing the binary variables to take on any real value between 0 and 1 . Finally, we let $\mathcal{F}_{L}$ denote the relaxation of $\mathcal{F}_{S}$ obtained by dropping the integrality restriction on $y$ as well.

We assume that the data is rational. Without loss of generality, we assume that $a_{i}>0$ for all $i \in N$, since if $a_{i}<0, x_{i}$ can be complemented and if $a_{i}=0, x_{i}$ can be dropped. We do not impose a sign restriction on the constant term $a_{0}$, as for the related separation and lifting problems that will be discussed in the following sections, this term may take on negative or nonnegative values. Throughout the paper, for a vector $v \in \mathbb{R}^{n}$, we let $v(S) \equiv \sum_{i \in S} v_{i}$ for $S \subseteq N$.

\section{Related work \& contributions of this paper}

Polyhedral structure of the 0-1 knapsack set, which is a restriction of $\mathcal{F}_{U}$, has been studied extensively; see $[3,16,25,23,27,24,15]$. Another set related to $\mathcal{F}_{U}$ is the $0-1$ knapsack set with single continuous variable, obtained by replacing $y$ with a nonnegative continuous variable, is studied in Marchand and Wolsey [20].

Magnanti et al. [19] study the facial structure of $\operatorname{conv}\left(\mathcal{F}_{S}\right)$ when $a_{0}=0$. They define an exponential class of valid inequalities, called the residual capacity inequalities, and show that the residual capacity inequalities and the constraints of $\mathcal{F}_{L}$ are sufficient to describe $\operatorname{conv}\left(\mathcal{F}_{S}\right)$. However, no exact polynomial-time separation algorithm for these inequalities was known until now. In Sect. 2 we present a linear-time algorithm for separating the residual capacity inequalities.

For $\mathcal{F}_{U}$ Brockmüller et al. [7] introduce the $c$-strong inequalities and characterize the necessary and sufficient conditions under which the $c$-strong inequalities are facetdefining. Recently, van Hoesel et al. [17] study $\mathcal{F}_{U}$ when $a_{0}=0$ as well. In Sect. 3 
we prove that the $c$-strong inequalities constitute all facet-defining inequalities of $\operatorname{conv}\left(\mathcal{F}_{U}\right)$ of the form $\sum_{i \in N} \pi_{i} x_{i} \leq \pi_{o}+y$ with integral coefficients. We show that the separation problem of $c$-strong inequalities is $\mathcal{N} \mathcal{P}$-hard and that it is sufficient to solve this separation problem over the subspace of fractional variables only. Furthermore, we introduce two classes of inequalities, both of which include the $c$-strong inequalities as a special case.

In Sect. 4 we provide a summary of computational studies with a branch-and-cut algorithm for multicommodity flow capacitated network design problems to test the effectiveness of the results presented here empirically.

\section{Splittable flow arc set}

\subsection{Optimization problem}

In order to motivate the separation problem of the splittable flow arc set $\mathcal{F}_{S}$, we start with the related optimization problem. Magnanti et al. [19] state that the optimization of a linear function over $F_{S}$ can be solved efficiently using an incremental strategy. Here we give a simple algorithm, which is used in Sect. 4 for approximate lifting of valid inequalities for $\mathcal{F}_{U}$. We consider a maximization problem and without loss of generality assume that the objective coefficient of the capacity variable $y$ is negative and by scaling -1 , since otherwise the problem is unbounded. So consider the problem

$$
\text { (SFP) } \quad \zeta=\max \left\{\sum_{i \in N} c_{i} x_{i}-y: \sum_{i \in N} a_{i} x_{i} \leq a_{0}+y,(x, y) \in \mathcal{D}_{S}\right\} .
$$

We may assume that $c_{i}>0$ for all $i \in N$, since otherwise given any optimal solution with $x_{i}>0$, there exists an optimal solution that is identical except that $x_{i}=0$. Suppose the variables are indexed so that $\frac{c_{1}}{a_{1}} \geq \frac{c_{2}}{a_{2}} \geq \cdots \geq \frac{c_{n}}{a_{n}}$, ties broken arbitrarily. Let $A_{i}=\sum_{h=1}^{i} a_{h}-a_{0}$ for $i \in N, A_{0}=-a_{0}$, and $k$ be the largest index with $\frac{c_{k}}{a_{k}} \geq 1$. If $\frac{c_{1}}{a_{1}}<1$, let $k=0$. Clearly, there exists an optimal solution to the linear programming relaxation of SFP with all positive $x_{i}$ in the above order and $y=A_{k}$. Then, by concavity of $\zeta(y)$, there exists an optimal solution $\left(x^{*}, y^{*}\right)$ to SFP such that $y^{*}=\left\lfloor A_{k}\right\rfloor$ or $y^{*}=\left\lceil A_{k}\right\rceil$. Hence the computational burden of finding an optimal solution to SFP is sorting the variables in nonincreasing order of $\frac{c_{i}}{a_{i}}$, which can be done in $O(n \log n)$.

Proposition 1. The optimization problem SFP can be solved in $O(n \log n)$.

In the light of polynomial equivalence of optimization and separation for a polyhedron [13], the separation problem of $\operatorname{conv}\left(\mathcal{F}_{S}\right)$ must also be solvable in polynomial time. In the next section, we show that $\operatorname{conv}\left(\mathcal{F}_{S}\right)$ can be separated in linear time.

\subsection{Separation problem}

For $S \subseteq N$ let $\eta=\left\lceil a(S)-a_{0}\right\rceil$ and $r=a(S)-a_{0}-\left\lfloor a(S)-a_{0}\right\rfloor$. Magnanti et al. [19] show that for any $S \subseteq N$ the residual capacity inequality

$$
\sum_{i \in S} a_{i}\left(1-x_{i}\right) \geq r(\eta-y)
$$


is valid for $\mathcal{F}_{S}$ when $a_{0}=0$. Inequality (2) is valid for $\mathcal{F}_{S}$ when $r=0$ or $y \geq \eta$ since $x_{i} \leq 1$ for all $i \in N$. To see that it is also valid otherwise, observe that $\sum_{i \in S} a_{i}\left(1-x_{i}\right) \geq$ $a(S)-a_{0}-y \geq \eta-(1-r)-y=(1-r)(\eta-1)+r \eta-y \geq(1-r) y+r \eta-y=r(\eta-y)$. The residual capacity inequality can also be viewed as a mixed-integer rounding inequality from a suitable relaxation of $\mathcal{F}_{S}$ [21].

Magnanti et al. [19] prove that the residual capacity inequalities together with the constraints of $\mathcal{F}_{L}$ are sufficient to describe $\operatorname{conv}\left(\mathcal{F}_{S}\right)$ when $a_{0}=0$. This result extends to the case when $a_{0} \neq 0$ as well. Since the constraints of $\mathcal{F}_{L}$ can be checked for violation simply by testing in linear time, the separation problem of $\operatorname{conv}\left(\mathcal{F}_{S}\right)$ reduces to, given a point $(\bar{x}, \bar{y}) \in \mathcal{F}_{L}$, either finding a residual capacity inequality violated by $(\bar{x}, \bar{y})$, or concluding that $(\bar{x}, \bar{y}) \in \operatorname{conv}\left(\mathcal{F}_{S}\right)$. Without loss of generality, we may assume that $\bar{y} \notin \mathbb{Z}$, because no $(\bar{x}, \bar{y}) \in \mathcal{F}_{L}$ with $\bar{y} \in \mathbb{Z}$ violates a residual capacity inequality as residual capacity inequalities are valid for $\mathcal{F}_{S}$. So we look for $S \subseteq N$ such that $\sum_{i \in S} a_{i}\left(1-\bar{x}_{i}\right)<r(\eta-\bar{y})$. We are interested in only $S$ with $r>0$, since no residual capacity inequality with $r=0$ is violated by $(\bar{x}, \bar{y}) \in \mathcal{F}_{L}$ as $\bar{x}_{i} \leq 1$ for all $i \in N$. Then, the separation problem can be formulated as

$$
\begin{aligned}
\varsigma=\min & \sum_{i \in N} a_{i}\left(1-\bar{x}_{i}\right) z_{i}-r(\eta-\bar{y}) \\
\text { s.t.: } & \sum_{i \in N} a_{i} z_{i}=a_{0}+(\eta-1)+r \\
& 0<r<1, \quad \eta \in \mathbb{Z}, \quad z_{i} \in\{0,1\} \quad i \in N,
\end{aligned}
$$

where $z \in\{0,1\}^{n}$ is the characteristic vector of $S$. If $\varsigma<0$, then the residual capacity inequality corresponding to an optimal $(z, \eta, r)$ is violated by $(\bar{x}, \bar{y})$, otherwise, no residual capacity inequality is violated by $(\bar{x}, \bar{y})$. SP1 is a nonlinear mixed-integer optimization problem, which is hard to solve in general.

Lemma 1. A point $(\bar{x}, \bar{y}) \in \mathcal{F}_{L}$ does not violate any residual capacity inequality (2) with $\eta \leq \bar{y}$ or $\eta \geq \bar{y}+1$.

Proof. Since residual capacity inequality (2) is the mixed-integer rounding inequality for the relaxation $\sum_{i \in S} a_{i}\left(1-x_{i}\right)+y \geq a(S)-a_{0}$, it is dominated by $\sum_{i \in S} a_{i}\left(1-x_{i}\right) \geq 0$ and $\sum_{i \in S} a_{i}\left(1-x_{i}\right)+y \geq a(S)-a_{0}$ unless $\eta-1<y<\eta$.

From Lemma 1 any residual capacity inequality violated by $(\bar{x}, \bar{y})$ has $\eta=\lceil\bar{y}\rceil$. Since $\bar{y} \notin \mathbb{Z}$, we have $\lfloor\bar{y}\rfloor=\eta-1$. After fixing $\eta$ to $\lceil\bar{y}\rceil$, the separation problem can be formulated as the following linear mixed 0-1 optimization problem

$$
\begin{aligned}
\varsigma=\min & \sum_{i \in N} a_{i}\left(1-\bar{x}_{i}\right) z_{i}-r(\lceil\bar{y}\rceil-\bar{y}) \\
\text { s.t.: } & \sum_{i \in N} a_{i} z_{i}=a_{0}+\lfloor\bar{y}\rfloor+r \\
& 0<r<1, \quad z_{i} \in\{0,1\} \quad i \in N .
\end{aligned}
$$


Eliminating the bounded continuous variable $r$, we rewrite the separation problem as a $0-1$ problem with two strict inequalities

$$
\begin{aligned}
\varsigma=\min & \sum_{i \in N} a_{i}\left(1-\bar{x}_{i}-\lceil\bar{y}\rceil+\bar{y}\right) z_{i}+(\lceil\bar{y}\rceil-\bar{y})\left(a_{0}+\lfloor\bar{y}\rfloor\right) \\
\text { s.t.: } & a_{0}+\lfloor\bar{y}\rfloor<\sum_{i \in N} a_{i} z_{i}<a_{0}+\lceil\bar{y}\rceil \\
& z_{i} \in\{0,1\} \quad i \in N .
\end{aligned}
$$

Next we show that in order to find a violated residual capacity inequality, it is sufficient to consider only variables with a negative coefficient in the objective function of SP3. Let $T \equiv\left\{i \in N: 1-\bar{x}_{i}<\lceil\bar{y}\rceil-\bar{y}\right\}$.

Lemma 2. If there exists a residual capacity inequality (2) violated by a point $(\bar{x}, \bar{y}) \in$ $\mathcal{F}_{L}$, then there exists one given by $S \subseteq T$.

Proof. Suppose the residual capacity inequality given by $C \subseteq N$ is violated by $(\bar{x}, \bar{y})$. Then, $a(C \cap T)+a(C \backslash T)=a_{0}+\lfloor\bar{y}\rfloor+r$. Consider the following two cases.

(1) $a(C \backslash T)<r$. In this case $a_{0}+\lfloor\bar{y}\rfloor<a(C \cap T)<a_{0}+\lceil\bar{y}\rceil$ and $C \cap T$ has an objective value in SP3 that is no more than that of $C$. So let $S=C \cap T$.

(2) $a(C \backslash T) \geq r$. In this case $a(C \cap T) \leq a_{0}+\lfloor\bar{y}\rfloor$. Therefore, the objective value for $C$ in SP3 is $\sum_{i \in C} a_{i}\left(1-\bar{x}_{i}-\lceil\bar{y}\rceil+\bar{y}\right)+(\lceil\bar{y}\rceil-\bar{y})\left(a_{0}+\lfloor\bar{y}\rfloor\right) \geq \sum_{i \in C} a_{i}\left(1-\bar{x}_{i}-\lceil\bar{y}\rceil+\bar{y}\right)+$ $(\lceil\bar{y}\rceil-\bar{y}) a(C \cap T)=\sum_{i \in C \cap T} a_{i}\left(1-\bar{x}_{i}\right)+\sum_{i \in C \backslash T} a_{i}\left(1-\bar{x}_{i}-\lceil\bar{y}\rceil+\bar{y}\right) \geq 0$. However, this contradicts the assumption that the residual capacity given by $C$ is violated.

Lemma 3. If $a(T) \leq a_{0}+\lfloor\bar{y}\rfloor$ or $a(T) \geq a_{0}+\lceil\bar{y}\rceil$, then there exists no residual capacity inequality violated by $(\bar{x}, \bar{y}) \in \mathcal{F}_{L}$.

Proof. Suppose $a(T) \leq a_{0}+\lfloor\bar{y}\rfloor$. Then, there exists no $S \subseteq T$ that satisfies the constraints of SP3 and therefore, from Lemma 2, there exists no residual capacity inequality that is violated by $(\bar{x}, \bar{y})$. Now suppose $a(T) \geq a_{0}+\lceil\bar{y}\rceil$ and, for contradiction, suppose there exists a set $S$ that gives a violated inequality. From Lemma 2, we may assume that $S \subseteq T$. The objective value for $S$ in SP3 is $\sum_{i \in S} a_{i}\left(1-\bar{x}_{i}-\lceil\bar{y}\rceil+\bar{y}\right)$ $+(\lceil\bar{y}\rceil-\bar{y})\left(a_{0}+\lfloor\bar{y}\rfloor\right) \geq \sum_{i \in T} a_{i}\left(1-\bar{x}_{i}-\lceil\bar{y}\rceil+\bar{y}\right)+(\lceil\bar{y}\rceil-\bar{y})\left(a_{0}+\lfloor\bar{y}\rfloor\right) \geq$ $\left(\lceil\bar{y}\rceil+a_{0}\right)(1-\lceil\bar{y}\rceil+\bar{y})-\left(a_{0}+\bar{y}\right)+(\lceil\bar{y}\rceil-\bar{y})\left(a_{0}+\lfloor\bar{y}\rfloor\right)=0$. This contradicts the assumption that the inequality is violated by $(\bar{x}, \bar{y})$.

\section{Separation Algorithm}

Since the residual capacity inequalities, together with the inequalities of $\mathcal{F}_{L}$ describe $\operatorname{conv}\left(\mathcal{F}_{S}\right)$, we have the following simple procedure for separating $(\bar{x}, \bar{y}) \in \mathcal{F}_{L} \backslash \mathcal{F}_{S}$ from $\operatorname{conv}\left(\mathcal{F}_{S}\right)$ : Let $T \equiv\left\{i \in N: \bar{x}_{i}>\bar{y}-\lfloor\bar{y}\rfloor\right\}$. If $a_{0}+\lfloor\bar{y}\rfloor<a(T)<a_{0}+\lceil\bar{y}\rceil$ and $\sum_{i \in T} a_{i}\left(1-\bar{x}_{i}-\lceil\bar{y}\rceil+\bar{y}\right)+(\lceil\bar{y}\rceil-\bar{y})\left(a_{0}+\lfloor\bar{y}\rfloor\right)<0$, then the inequality $\sum_{i \in T} a_{i}\left(1-x_{i}\right) \geq$ $r(\eta-y)$ is violated by $(\bar{x}, \bar{y})$. Otherwise, there exists no residual capacity inequality violated by $(\bar{x}, \bar{y})$. Clearly, this procedure can be performed in linear time.

Theorem 1. The separation problem for the residual capacity inequalities (2) can be solved in $O(n)$. 


\section{Unsplittable flow arc set}

\subsection{Optimization problem}

Even though our ultimate goal is to find strong valid inequalities for the unsplittable flow arc set $\mathcal{F}_{U}$, it is helpful to study the maximization of a linear function over $\mathcal{F}_{U}$ first. As for $\mathcal{F}_{S}$ we may assume that the objective coefficient of the capacity variable $y$ is -1 and state the problem as

$$
\xi=\max \left\{\sum_{i \in N} c_{i} x_{i}-y: \sum_{i \in N} a_{i} x_{i} \leq a_{0}+y, \quad(x, y) \in \mathcal{D}_{U}\right\} .
$$

UFP is a relaxation of the more familiar 0-1 knapsack problem. Although the formulation of UFP is quite similar to the formulation of the knapsack problem, its structure is significantly different from the latter. Below we present properties of optimal solutions of UFP that will be useful when studying $\operatorname{conv}\left(\mathcal{F}_{U}\right)$ in Sect. 3.2.

Proposition 2. UFP has an optimal solution $\left(x^{*}, y^{*}\right)$ such that

$$
x_{i}^{*}=\left\{\begin{array}{ll}
1 & \text { if } c_{i} \geq\left\lceil a_{i}\right\rceil \\
0 & \text { if } c_{i} \leq\left\lfloor a_{i}\right\rfloor
\end{array} \text { for } i \in N .\right.
$$

Proof. For $S \subseteq N$ let $\xi(S)$ be the maximum value of the objective of UFP when $x_{k}=1$ for all $k \in S$, and $x_{k}=0$ otherwise, i.e., $\xi(S)=c(S)-\left\lceil a(S)-a_{0}\right\rceil$. Suppose $i \notin S$. Then $\xi(S)-\xi(S \cup i)=\left\lceil a(S)+a_{i}-a_{0}\right\rceil-\left\lceil a(S)-a_{0}\right\rceil-c_{i}$. Since for any $a, b \in \mathbb{R}$, we have $\lceil a\rceil+\lfloor b\rfloor \leq\lceil a+b\rceil \leq\lceil a\rceil+\lceil b\rceil$, it follows that

$$
\xi(S)-\xi(S \cup i)\left\{\begin{array}{l}
\leq 0 \text { if } c_{i} \geq\left\lceil a_{i}\right\rceil \\
\geq 0 \text { if } c_{i} \leq\left\lfloor a_{i}\right\rfloor
\end{array}\right.
$$

Due to Proposition 2, all binary variables except the ones with $\left\lfloor a_{i}\right\rfloor<c_{i}<\left\lceil a_{i}\right\rceil$ can be eliminated from UFP since optimal values for them can be determined a priori.

Corollary 1. UFP can be solved in $O(n)$ if either $c_{i} \in \mathbb{Z}$ or $a_{i} \in \mathbb{Z}$ for all $i \in N$.

Theorem 2. UFP is $\mathcal{N} \mathcal{P}$-hard for any fixed value of $a_{0}$.

Proof. The proof is by reduction from PARTITION [11]. Given a set $N$ and weights $a_{i}$ $i \in N$ with $a(N)=2$, PARTITION is the question whether there exists $S \subset N$ such that $a(S)=1$. Let $a_{0}$ be fixed to $\bar{a}_{0}$ and let $\bar{f}_{0}=\bar{a}_{0}-\left\lfloor\bar{a}_{0}\right\rfloor$. In order to answer PARTITION, we construct the following instance of UFP with $a_{0}=\bar{a}_{0}$

$$
\begin{aligned}
\varsigma=\max & \sum_{i \in N} a_{i} x_{i}+3 \alpha x_{o}-\alpha y \\
\text { s.t.: } & \sum_{i \in N} a_{i} x_{i}+\left(\epsilon+\bar{f}_{0}(1+\epsilon)\right) x_{o} \leq(1+\epsilon)\left(\bar{a}_{0}+y\right) \\
& x_{i} \in\{0,1\} i \in N, x_{o} \in\{0,1\}, y \in \mathbb{Z},
\end{aligned}
$$


where $\alpha>1$ and $1>\epsilon>0$. After dividing the objective by $\alpha$ and the constraint by $1+\epsilon$, since $\left\lceil\frac{\epsilon}{1+\epsilon}+\bar{f}_{0}\right\rceil<3$, from the proof of Proposition 2 we see that $x_{o}=1$ and hence $y \geq\left\lceil-\bar{a}_{0}\right\rceil+1$ in every optimal solution to UFP. Also since $a(N)+\epsilon+\bar{f}_{0}(1+\epsilon)<$ $(1+\epsilon)\left(\bar{f}_{0}+2\right)$, we have $y \leq\left\lceil-\bar{a}_{0}\right\rceil+2$ in any optimal solution. The objective value $\varsigma(y)$ as a function of $y$ satisfies the following: $\varsigma\left(\left\lceil-\bar{a}_{0}\right\rceil+1\right) \leq 1+2 \alpha+\alpha\left\lfloor\bar{a}_{0}\right\rfloor$ and $\varsigma\left(\left\lceil-\bar{a}_{0}\right\rceil+2\right) \leq 2+\alpha+\alpha\left\lfloor\bar{a}_{0}\right\rfloor$. Let $S^{*}$ be the index set of binary variables at value 1 in an optimal solution. Since $\alpha>1, \varsigma=1+2 \alpha+\alpha\left\lfloor\bar{a}_{0}\right\rfloor$ if and only $a\left(S^{*}\right)=1$. Hence, the PARTITION problem has an affirmative answer if and only if the optimal value of UFP equals $1+2 \alpha+\alpha\left\lfloor\bar{a}_{0}\right\rfloor$.

Remark 1. Theorem 2 states that UFP remains $\mathcal{N} \mathcal{P}$-hard for any fixed value of $a_{0}$. In Sect. 3.2 we will see that $a_{0}$ may take on negative values in the separation problem of $c$-strong inequalities.

Now we give a canonical form of UFP. Without loss of generality, we assume that $\left\lfloor a_{i}\right\rfloor<c_{i}<\left\lceil a_{i}\right\rceil$ for all $i \in N$ for UFP, since all other variables can be eliminated from the problem by Proposition 2. Then we can further simplify the problem into one where the data consists of only the fractional parts of $a_{i}$ and $c_{i}$. Let $f_{0}=a_{0}-\left\lfloor a_{0}\right\rfloor$ and for $i \in N$ let $f_{i}=a_{i}-\left\lfloor a_{i}\right\rfloor$ and $r_{i}=c_{i}-\left\lfloor c_{i}\right\rfloor$ and define

$$
\left(\mathrm{UFP}_{f}\right) \quad \xi_{f}=\max \left\{\sum_{i \in N} r_{i} x_{i}-y: \sum_{i \in N} f_{i} x_{i} \leq f_{0}+y,(x, y) \in \mathcal{D}_{U}\right\} .
$$

Proposition 3. UFP $_{f}$ is equivalent to UFP in the sense that $S \subseteq N$ maximizes UFP if and only if it maximizes $U F P_{f}$ and $\xi_{f}=\xi-\left\lfloor a_{0}\right\rfloor$.

Proof. For $S \subseteq N$ let $\xi(S)=c(S)-\left\lceil a(S)-a_{0}\right\rceil$. Then

$$
\begin{aligned}
\xi(S) & =\sum_{i \in S}\left\lfloor c_{i}\right\rfloor+r(S)-\sum_{i \in S}\left\lfloor a_{i}\right\rfloor+\left\lfloor a_{0}\right\rfloor-\left\lceil f(S)-f_{0}\right\rceil \\
& =r(S)+\left\lfloor a_{0}\right\rfloor-\left\lceil f(S)-f_{0}\right\rceil=\xi_{f}(S)+\left\lfloor a_{0}\right\rfloor .
\end{aligned}
$$

\section{Optimization algorithm}

Next we give a pseudo polynomial algorithm for solving $\mathrm{UFP}_{f}$, which is used to show strongly polynomial-time lifting valid inequalities in Theorem 5. Let $\lambda$ be a common multiple of the denominators of the rational numbers $f_{i} i=0,1, \ldots, n$. By multiplying $f_{i}$ with $\lambda$, the constraint of $\mathrm{UFP}_{f}$ can be written with integral coefficients only. Observe that since $f_{i}<1, y$ can take on a value at most $\left\lceil f(N)-f_{0}\right\rceil \leq n$ in an optimal solution to $\mathrm{UFP}_{f}$. For $v \in\left\{0,1, \ldots,\left\lceil f(N)-f_{0}\right\rceil\right\}$, consider an optimal solution $x^{*}$ to the $0-1$ knapsack problem

$$
(\operatorname{KP} 1(v)) \quad \varsigma(v)=\max \left\{\sum_{i \in N} r_{i} x_{i}: \sum_{i \in N} \lambda f_{i} x_{i} \leq \lambda\left(f_{0}+v\right), x \in\{0,1\}^{n}\right\} .
$$

Since $r_{i}>0$ and $f_{i}<1$ for all $i \in N$, we have $\lambda f_{0}+\lambda(\nu-1)<\sum_{i \in N} \lambda f_{i} x_{i}^{*} \leq \lambda f_{0}+\lambda \nu$. Hence, $\xi_{f}=\max _{v \in\left\{0,1, \ldots,\left\lceil f(N)-f_{0}\right\rceil\right\}}\{\zeta(\nu)-v\}$. Notice that all of these related, at most 
$n+1$, knapsack problems can be solved in a total of $O\left(\lambda n^{2}\right)$ by dynamic programming, since when solving $\mathrm{KP} 1(n)$, we already complete the computations required for solving $\mathrm{KP} 1(v)$ for all $v \in\{0,1, \ldots, n-1\}$.

Alternatively, let $\mu$ be a common multiple of the denominators of the fractional numbers $r_{i} i=1,2, \ldots, n$. Instead of solving $\mathrm{KP} 1(v)$, we may solve the dual knapsack problem

$$
(\operatorname{KP} 2(t)) \quad \omega(t)=\min \left\{\sum_{i \in N} f_{i} x_{i}: \sum_{i \in N} \mu r_{i} x_{i} \geq t, x \in\{0,1\}^{n}\right\}
$$

so that $\zeta(v)=\omega(t)$ if $\omega(t) \leq \lambda\left(f_{0}+v\right)<\omega(t+1)$. Since KP2(t) is infeasible for $t \geq \mu n>\mu r(N), \varsigma(v)$ for all $v \in\{0,1, \ldots, n\}$ can be computed in $O\left(\mu n^{2}\right)$.

Theorem 3. UFP can be solved in $O\left(\min \{\lambda, \mu\} n^{2}\right)$.

\subsection{Valid inequalities}

In this section we discuss three classes of valid inequalities for $\mathcal{F}_{U}$. The first class is the $c-$ strong inequalities introduced by Brockmüller et al. [7]. The next two classes are new and both of them subsume the $c$-strong inequalities. Before describing specific valid inequalities, we present some general properties of $\operatorname{conv}\left(\mathcal{F}_{U}\right)$ that will be useful in the analysis of those inequalities. First of all, it is easy to check that the convex hull of $\mathcal{F}_{U}$, $\operatorname{conv}\left(\mathcal{F}_{U}\right)$, is full-dimensional and inequalities $x_{i} \geq 0$ and $x_{i} \leq 1$ for all $i \in N$ are facet-defining for $\operatorname{conv}\left(\mathcal{F}_{U}\right)$. We call these inequalities as the trivial valid inequalities of $\operatorname{conv}\left(\mathcal{F}_{U}\right)$. The next proposition provides bounds on the coefficients of non-trivial facet-defining valid inequalities of $\operatorname{conv}\left(\mathcal{F}_{U}\right)$.

Proposition 4. [2,17] Every non-trivial facet-defining inequality $\sum_{i \in N} \pi_{i} x_{i} \leq \pi_{o}+y$ of $\operatorname{conv}\left(\mathcal{F}_{U}\right)$ has $\left\lfloor a_{i}\right\rfloor \leq \pi_{i} \leq\left\lceil a_{i}\right\rceil$ for all $i \in N$ and $\pi_{o} \geq\left\lfloor a_{0}\right\rfloor . \pi_{o}=\max \left\{\sum_{i \in N} \pi_{i} x_{i}-y\right.$ : $\left.(x, y) \in \mathcal{F}_{U}\right\}$.

As before let $f_{i}=a_{i}-\left\lfloor a_{i}\right\rfloor$ for $i=0,1, \ldots, n$ and define

$$
\mathcal{F}_{U f} \equiv\left\{(x, y) \in \mathcal{D}_{U}: \sum_{i \in N} f_{i} x_{i} \leq f_{0}+y\right\} .
$$

Proposition 5. An inequality $\sum_{i \in N} \pi_{i} x_{i} \leq \pi_{o}+y$ with $\left\lfloor a_{i}\right\rfloor \leq \pi_{i} \leq\left\lceil a_{i}\right\rceil$ is valid for $\mathcal{F}_{U}$ if and only if $\sum_{i \in N}\left(\pi_{i}-\left\lfloor\pi_{i}\right\rfloor\right) x_{i} \leq \pi_{o}-\left\lfloor a_{0}\right\rfloor+y$ is valid for $\mathcal{F}_{U f}$.

Proof. Follows from Proposition 3.

Remark 2. From Proposition 4 when looking for strong valid inequalities for $\mathcal{F}_{U}$, we can restrict our attention to inequalities $\sum_{i \in N} \pi_{i} x_{i} \leq \pi_{o}+y$ with $\left\lfloor a_{i}\right\rfloor \leq \pi_{i} \leq\left\lceil a_{i}\right\rceil$ for all $i \in N$. But then, from Proposition 5, instead of working with $\mathcal{F}_{U}$, we can work with $\mathcal{F}_{U f}$ defined using the fractional parts of the data. 


\section{Nonrestrictive assumption}

Due to Propositions 4 and 5, without loss of generality, we assume that $0<a_{i}<1$ for all $i \in N$ and $0 \leq a_{0}<1$; so $\mathcal{F}_{U}=\mathcal{F}_{U f}$ for the rest of the paper. Consequently, from Proposition 4, $0 \leq \pi_{i} \leq 1$ for all $i \in N$ for all non-trivial facet-defining inequalities of $\operatorname{conv}\left(\mathcal{F}_{U}\right)$.

\subsection{1. $c$-strong inequalities}

For $S \subseteq N$ let $c_{S}=|S|-\left\lceil a(S)-a_{0}\right\rceil . S$ is said to be maximal $c$-strong if $c_{S \backslash\{i\}}=c_{S}$ for all $i \in S$ and $c_{S \cup\{i\}}=c_{S}+1$ for all $i \in N \backslash S$. Brockmüller et al. [7] show that for any $S \subseteq N$ the $c$-strong inequality

$$
\sum_{i \in S} x_{i} \leq c_{S}+y
$$

is valid for $\mathcal{F}_{U}$ when $a_{0}=0$. A $c$-strong inequality is facet-defining for $\operatorname{conv}\left(\mathcal{F}_{U}\right)$ if and only if $S$ is maximal $c$-strong.

Theorem 4. The maximal c-strong inequalities (3) constitute all facet-defining inequalities of $\operatorname{conv}\left(\mathcal{F}_{U}\right)$ of the form $\sum_{i \in N} \pi_{i} x_{i} \leq \pi_{o}+y$ with integral coefficients.

Proof. Let $\sum_{i \in N} \pi_{i} x_{i} \leq \pi_{o}+y$ be a facet-defining inequality of $\operatorname{conv}\left(\mathcal{F}_{U}\right)$ with integral coefficients. From Proposition 4, it follows that $\pi_{i} \in\{0,1\}$ for $i \in N$. Let $S=\left\{i \in N: \pi_{i}=1\right\}$. From Proposition 2, we have that $\pi_{0}=c_{S}$.

In the next sections, we show that $\operatorname{conv}\left(\mathcal{F}_{U}\right)$ does have facet-defining inequalities $\sum_{i \in N} \pi_{i} x_{i} \leq \pi_{o}+y$ with fractional coefficients.

\section{Separation}

Given a point $(\bar{x}, \bar{y})$, there exists a $c$-strong inequality violated by $(\bar{x}, \bar{y})$ if and only if $\exists S \subseteq N$ such that $\sum_{i \in S} \bar{x}_{i}-c_{S}>\bar{y}$. Then, a $c$-strong inequality is violated if and only if $\max _{S \subseteq N}\left\{\sum_{i \in S} \bar{x}_{i}-\left\lfloor a_{0}+\sum_{i \in S}\left(1-a_{i}\right)\right\rfloor\right\}=\max \left\{\sum_{i \in N} \bar{x}_{i} z_{i}-w: \sum_{i \in N}\left(1-a_{i}\right) z_{i}\right.$ $\left.+a_{0}+1 / \lambda \leq w,(z, w) \in \mathcal{D}_{U}\right\}+1>\bar{y}$, where $\lambda$ is the least common multiple of the denominators of the rational numbers $\left(1-a_{i}\right)$ and $a_{0}$. From Theorem 2 the last maximization problem with the constant term $-a_{0}-1 / \lambda$ is $\mathcal{N} \mathcal{P}$-hard.

Although the separation problem of $c$-strong inequalities is $\mathcal{N} \mathcal{P}$-hard, from Proposition 2, it has an optimal solution $\left(z^{*}, w^{*}\right)$ such that $z_{i}^{*}=1$ if $\bar{x}_{i}=1$ and $z_{i}^{*}=0$ if $\bar{x}_{i}=0$. Therefore, we can fix such variables to their optimal values and solve the separation problem over $i \in N$ such that $0<\bar{x}_{i}<1$, which in practice can be done very efficiently even by enumeration, as most variables take on values either 0 or 1 in the LP relaxations of network design problems.

\subsection{2. $k$-split c-strong inequalities}

In this section we describe new valid inequalities for $\mathcal{F}_{U}$ that are motivated by Proposition 2. An inequality $\sum_{i \in N} \pi_{i} x_{i} \leq \pi_{o}+y$ is valid for $\mathcal{F}_{U}$ if and only if 
$\max \left\{\sum_{i \in N} \pi_{i} x_{i}-y:(x, y) \in \mathcal{F}_{U}\right\} \leq \pi_{o}$. As shown in Sect. 3.1 solving this maximization problem is $\mathcal{N} \mathcal{P}$-hard. However, if the maximum of $\sum_{i \in N} \pi_{i} x_{i}-y$ over a suitable relaxation of $\mathcal{F}_{U}$ is no more than $\pi_{o}$, then we can deduce that $\sum_{i \in N} \pi_{i} x_{i} \leq \pi_{o}+y$ is valid for this relaxation and hence for $\mathcal{F}_{U}$. The relaxation of $\mathcal{F}_{U}$ that we consider for this purpose is obtained by splitting the integer capacity variable. In a $k$-split relaxation, the capacity variable $y$ is allowed to take values that are integer multiples of $1 / k$, where $k$ is a positive integer. Let $\mathcal{F}_{U}^{k} \equiv\left\{\sum_{i \in N} a_{i} x_{i} \leq a_{0}+z / k,(x, z) \in \mathcal{D}_{U}\right\}$. Thus we define an infinite set of relaxations for $\mathcal{F}_{U}$ with $\operatorname{conv}\left(\mathcal{F}_{U}^{1}\right)=\operatorname{conv}\left(\mathcal{F}_{U}\right)$ and $\lim _{k \rightarrow \infty} \operatorname{conv}\left(\mathcal{F}_{U}^{k}\right)=\mathcal{F}_{L}$. The last equation follows from the fact that $x \in\{0,1\}^{n}$ for all extreme points $(x, y)$ of $\mathcal{F}_{L}$. For a given instance of $\mathcal{F}_{U}$, since there exists a finite $k$ for which $k c_{i} \leq\left\lfloor k a_{i}\right\rfloor$ if $c_{i} \leq a_{i}$ and $k c_{i} \geq\left\lceil k a_{i}\right\rceil$ if $c_{i} \geq a_{i}$ for all $i \in N$, from Proposition 2, there exists a $k$-split relaxation of $\mathcal{F}_{U}$, for which the optimization problem is trivial to solve and hence the validity of a given inequality $\sum_{i \in N} \pi_{i} x_{i} \leq \pi_{o}+y$ can be checked easily for this relaxation. Alternatively, for each $k$ we can define an inequality that can be easily verified to be valid for the corresponding $k$-split relaxation.

Proposition 6. Any inequality $\sum_{i \in N} \pi_{i} x_{i} \leq \pi_{o}+k y$ with $k$ positive integer and $\pi_{i} \leq$ $\left\lfloor k a_{i}\right\rfloor$ or $\pi_{i} \geq\left\lceil k a_{i}\right\rceil$ for all $i \in N$ is valid for $\mathcal{F}_{U}$ for $\pi_{o}=\pi(S)-\left\lceil k a(S)-k a_{0}\right\rceil$, where $S=\left\{i \in N: \pi_{i} \geq\left\lceil k a_{i}\right\rceil\right\}$.

Proof. The proof is an immediate consequence of Proposition 2.

$$
\begin{aligned}
\pi_{o} & =\pi(S)-\left\lceil k a(S)-k a_{0}\right\rceil \\
& =\max \left\{\sum_{i \in N} \pi_{i} x_{i}-z: \sum_{i \in N} k a_{i} x_{i} \leq k a_{0}+z, \quad(x, z) \in \mathcal{D}_{U}\right\} \\
& \geq \max \left\{\sum_{i \in N} \pi_{i} x_{i}-k y: \sum_{i \in N} a_{i} x_{i} \leq a_{0}+y, \quad(x, y) \in \mathcal{D}_{U}\right\} \quad(k y=z) .
\end{aligned}
$$

Then for any positive integer $k$ and any $S \subseteq N$, we can define strong valid inequalities by letting $\pi_{i} i \in N$ equal either $\left\lfloor k a_{i}\right\rfloor$ or $\left\lceil k a_{i}\right\rceil$. Let $c_{S}^{k}=\sum_{i \in S}\left\lceil k a_{i}\right\rceil-\left\lceil k a(S)-k a_{0}\right\rceil$ and define the $k$-split c-strong inequality as

$$
\sum_{i \in S}\left\lceil k a_{i}\right\rceil x_{i}+\sum_{i \in N \backslash S}\left\lfloor k a_{i}\right\rfloor x_{i} \leq c_{S}^{k}+k y .
$$

Observe that $k$-split $c$-strong inequality (4) is a $c$-strong inequality for $\mathcal{F}_{U}^{k}$. Therefore, since $\mathcal{F}_{U}^{k}$ is a relaxation of $\mathcal{F}_{U}$, a necessary condition for inequality (4) to be facet-defining for $\mathcal{F}_{U}$ is that $S$ is maximal $c$-strong in the $k$-split relaxation $\mathcal{F}_{U}^{k}$. Recall that $a_{i}<1$ without loss of generality. Below we give a sufficient condition for the $k$-split $c$-strong inequality to be facet-defining for $\mathcal{F}_{U}$. As the example in Sect. 3.2.4 illustrates, $k$-split $c$-strong inequalities may be facet-defining more generally.

Proposition 7. The $k$-split c-strong inequality (4) is facet-defining for $\operatorname{conv}\left(\mathcal{F}_{U}\right)$ if (i) $S$ is maximal c-strong in the $k$-split relaxation, (ii) $f_{S}>(k-1) / k$ and $a_{0} \geq 0$, (iii) $a_{i}>f_{S}$ for all $i \in S, a_{i}<1-f_{S}$ for all $i \in N \backslash S$, where $f_{S}=a(S)-a_{0}-$ $\left\lfloor a(S)-a_{0}\right\rfloor$. 
Proof. Let $(C, z)$ denote a point $(x, z) \in \mathbb{B}^{n} \times \mathbb{Z}$ where $x_{i}=1$ for all $i \in C$ and $x_{i}=0$ otherwise. Consider the following $n+1$ affinely independent points of $\mathcal{F}_{U}^{k}$ :

$$
\begin{aligned}
& \left(S,\left\lceil k\left(a(S)-a_{0}\right)\right\rceil\right) \text { if } c_{S}^{k} \neq 0,(\emptyset, 0) \text { if } c_{S}^{k}=0 ; \\
& \left(S \backslash\{i\},\left\lceil k\left(a(S)-a_{i}-a_{0}\right)\right\rceil\right) \text { for } i \in S ; \\
& \left(S \cup\{i\},\left\lceil k\left(a(S)+a_{i}-a_{0}\right)\right\rceil\right) \text { for } i \in N \backslash S .
\end{aligned}
$$

Since $S$ is maximal $c$-strong in $k$-split relaxation, we have $\left\lceil k\left(a(S)-a_{0}\right)\right\rceil=\lceil k(a(S)-$ $\left.\left.a_{i}-a_{0}\right)\right\rceil+\left\lceil k a_{i}\right\rceil$ for all $i \in S$ and $\left\lceil k\left(a(S)-a_{0}\right)\right\rceil=\left\lceil k\left(a(S)+a_{i}-a_{0}\right)\right\rceil+\left\lfloor k a_{i}\right\rfloor$ for all $i \in N \backslash S$. Therefore, after replacing $z$ with $y=z / k$, these points satisfy the $k$-split $c$-strong inequality (4) at equality. To complete the proof, it is enough to show that $z / k$ is integer for the points above. For any $w \in \mathbb{R}$, let $f_{w}=w-\lfloor w\rfloor$. If $k f_{w}>k-1$, then $\left\lceil k f_{w}\right\rceil=k$ since $f_{w}<1$, so $\lceil k w\rceil=k\lfloor w\rfloor+\left\lceil k f_{w}\right\rceil=k\lceil w\rceil$. Thus, by part (ii) of the proposition, for the first point $\left\lceil k\left(a(S)-a_{0}\right)\right\rceil / k=\left\lceil a(S)-a_{0}\right\rceil$. Similarly, by part (iii), $\left\lceil k\left(a(S)-a_{i}-a_{0}\right)\right\rceil / k=\left\lfloor a(S)-a_{0}\right\rfloor$ for $i \in S$ and $\left\lceil k\left(a(S)+a_{i}-a_{0}\right)\right\rceil / k=\left\lceil a(S)-a_{0}\right\rceil$ for $i \in N \backslash S$ follow.

\subsubsection{Lifted knapsack cover inequalities}

Let $N_{0}$ and $N_{1}$ be two disjoint subsets of $N$ and $v$ be a nonnegative integer. Consider the $0-1$ knapsack set $\mathcal{F}_{U}\left(v, N_{0}, N_{1}\right)$ obtained by projecting the capacity variable $y$ to $v$, all binary variables indexed with $N_{0}$ to 0 and all binary variables indexed with $N_{1}$ to 1 , i.e., $\mathcal{F}_{U}\left(v, N_{0}, N_{1}\right) \equiv\left\{(x, y) \in \mathcal{F}_{U}: y=v, x_{i}=0\right.$ for all $i \in N_{0}$ and $x_{i}=1$ for all $\left.i \in N_{1}\right\}$. For this projection $C \equiv N \backslash\left(N_{0} \cup N_{1}\right)$ is called a cover if $r=$ $a(C)+a\left(N_{1}\right)-a_{0}-v>0$. $C$ is said to be a minimal cover if $a_{i} \geq r$ for all $i \in C$.

The knapsack cover inequality $\sum_{i \in C} x_{i} \leq|C|-1$ is well-known to be facetdefining for $\operatorname{conv}\left(\mathcal{F}_{U}\left(v, N_{0}, N_{1}\right)\right)$ if and only if $C$ is a minimal cover [22]. By lifting the knapsack cover inequalities of minimal covers with the projected variables, one can obtain facet-defining inequalities of $\operatorname{conv}\left(\mathcal{F}_{U}\right)$. One practical way of lifting inequalities is sequential lifting, in which projected variables are introduced to an inequality one at a time in some sequence. Van Hoesel et al. [17] have independently lifted knapsack cover inequalities to get strong valid inequalities for $\mathcal{F}_{U}$ as well. Here we show that given a minimal cover, a lifted cover inequality can be constructed in $O\left(n^{3}\right)$ if the capacity variable $y$ is lifted first. We further show that inequalities obtained in this manner subsume all $c$-strong inequalities.

Now we describe the lifting procedure. We introduce the capacity variable $y$ to the cover inequality first. Let $\mathcal{F}_{U}\left(N_{0}, N_{1}\right) \equiv\left\{(x, y) \in \mathcal{F}_{U}: x_{i}=0\right.$ for all $i \in N_{0}$ and $x_{i}=1$ for all $\left.i \in N_{1}\right\}$ and $C$ be a cover. Inequality $\sum_{i \in C} x_{i}+\alpha(v-y) \leq|C|-1$ is valid for $\mathcal{F}_{U}\left(N_{0}, N_{1}\right)$ if and only if

$$
\alpha \leq \bar{\alpha} \equiv \min \left\{\frac{|C|-1-\sum_{i \in C} x_{i}}{v-y}: y<v,(x, y) \in \mathcal{F}_{U}\left(N_{0}, N_{1}\right)\right\}
$$

and

$$
\alpha \geq \underline{\alpha} \equiv \max \left\{\frac{\sum_{i \in C} x_{i}-|C|+1}{y-v}: y>v,(x, y) \in \mathcal{F}_{U}\left(N_{0}, N_{1}\right)\right\} .
$$


If $C$ is a minimal cover and $\alpha$ equals either $\underline{\alpha}$ or $\bar{\alpha}$, then $\sum_{i \in C} x_{i}+\alpha(v-y) \leq|C|-1$ is facet-defining for $\operatorname{conv}\left(\mathcal{F}_{U}\left(N_{0}, N_{1}\right)\right)$, which follows from Wolsey [26]. The existence of a valid lifting coefficient $\alpha$ follows from the next proposition.

Proposition 8. [2,17] For any cover $C, \underline{\alpha} \leq 1 \leq \bar{\alpha}$ holds.

Note that $\underline{\alpha}=1 /\left(\left\lceil a(C)+a\left(N_{1}\right)-a_{0}\right\rceil-v\right)=1 /\lceil r\rceil$, which is computed in linear time for any cover. For a minimal cover $C$, since $0<r \leq a_{i}<1$, we have $\underline{\alpha}=1$. The upper bound $\bar{\alpha}$ can be computed efficiently as well: Suppose $a_{1} \leq a_{2} \leq \cdots \leq a_{|C|}$. Let $A_{0}=a\left(N_{1}\right)-a_{0}$ and $A_{i}=A_{i-1}+a_{i}$ for $i=1,2, \ldots,|C|$. Since the coefficients in the cover inequality are the same, we have

$$
\bar{\alpha}=\min _{i \in\{0,1, \ldots,|C|-2\}}\left\{\frac{|C|-1-i}{v-\left\lceil A_{i}\right\rceil}:\left\lceil A_{i}\right\rceil<v\right\} .
$$

Therefore $\bar{\alpha}$ is computed by selecting the minimum of at most $|C|-1$ terms after sorting $a_{i} i \in C$ in nondecreasing order, which can be done in $O(n \log n)$.

Next we introduce the projected binary variables to inequality $\sum_{i \in C} x_{i}+\alpha(v-y) \leq$ $|C|-1$ one at a time in some arbitrary sequence. As shown in the example in Sect. 3.2.4, different sequences may lead to different lifted inequalities. Let $L_{0} \subseteq N_{0}$ and $L_{1} \subseteq N_{1}$ be the index sets of variables that are already used in lifting and the current lifted inequality be

$$
\sum_{i \in C} x_{i}+\sum_{i \in L_{0}} \alpha_{i} x_{i}+\sum_{i \in L_{1}} \alpha_{i}\left(1-x_{i}\right)+\alpha(v-y) \leq|C|-1 .
$$

Then the lifting coefficient of a variable $x_{k}, k \in\left(N_{0} \backslash L_{0}\right) \cup\left(N_{1} \backslash L_{1}\right)$ is computed by solving the lifting problem

$$
\alpha_{k}=|C|-1-\max \sum_{i \in C} x_{i}+\sum_{i \in L_{0}} \alpha_{i} x_{i}+\sum_{i \in L_{1}} \alpha_{i}\left(1-x_{i}\right)+\alpha(v-y)
$$

(BLP)

$$
\begin{array}{cl}
\text { s.t.: } & \sum_{i \in C \cup L_{0} \cup L_{1}} a_{i} x_{i} \leq a_{0}-a\left(N_{1} \backslash L_{1}\right) \mp a_{k}+y \\
& x_{i} \in\{0,1\} i \in C \cup L_{0} \cup L_{1}, \quad y \in \mathbb{Z} .
\end{array}
$$

In the rhs of the constraint of BLP, we have $-a_{k}$ if $k \in N_{0}$ and $+a_{k}$ if $k \in N_{1}$.

Proposition 9. The maximal c-strong inequalities are equivalent to the lifted minimal cover inequalities with $\alpha=\underline{\alpha}$.

Proof. Let $S$ be maximal $c$-strong. Then the corresponding $c$-strong inequality $\sum_{i \in S} x_{i}$ $\leq c_{S}+y$ is facet-defining for $\operatorname{conv}\left(\mathcal{F}_{U}\right) . S$ is a minimal cover with $v=\left\lfloor a(S)-a_{0}\right\rfloor$, $N_{0}=N \backslash S$ and $N_{1}=\emptyset$. Consider the cover inequality lifted with the capacity variable using $\alpha=\underline{\alpha}=1, \sum_{i \in S} x_{i} \leq|S|-1-v+y$. Since $a_{i}<1$ and since $S$ is maximal $c$-strong, we have $\left\lfloor a(S)-a_{0}\right\rfloor<a(S)-a_{0}$, it follows that $|S|-1-v=c_{S}$. Since the $c$-strong inequality is facet-defining, the lifting coefficients of all of the projected binary variables must be 0 . Thus, the maximal $c$-strong inequality is indeed a lifted 
minimal cover inequality. The other direction follows from Theorem 4 since $\underline{\alpha}=1$ and hence the coefficients of the lifted cover inequality are integer.

Remark 3. The proof of Proposition 9 also shows that projecting binary variables to 1 does not lead to new inequalities when $\underline{\alpha}$ is used as the lifting coefficient for the capacity variable, because by Proposition 9 and Theorem 4 all facet-defining inequalities $\sum_{i \in N} \pi_{i} x_{i} \leq \pi_{o}+y$ of $\operatorname{conv}\left(\mathcal{F}_{U}\right)$ with integer coefficients can be obtained by lifting minimal cover inequalities using $\alpha=\underline{\alpha}$ and $N_{1}=\emptyset$, and letting $N_{1} \neq \varnothing$ does not lead to fractional lifting coefficients. So when $\alpha=\underline{\alpha}$, the lifted minimal cover inequalities take the simple form $\sum_{i \in C} x_{i} \leq|C|-1-v+y=c_{C}+y$.

Lemma 4. If $C$ is a minimal cover and $\alpha=\bar{\alpha}$, then the lifting coefficients of inequality (5) satisfy $\alpha_{i} \leq|C|-1$ for all $i \in N_{0}$ and $-\alpha_{i} \leq|C|-1$ for all $i \in N_{1}$.

Proof. From the definition of $\bar{\alpha}$, observe that $\bar{\alpha} \leq|C|-1$ with $y=v-1$. Since $C$ is a minimal cover, the lifted inequality is facet-defining for $\operatorname{conv}\left(\mathcal{F}_{U}\right)$. Then from Proposition 4, since $0<a_{i}<1$, we have $\alpha_{i} \leq \bar{\alpha}$ for $i \in N_{0}$ and $-\alpha_{i} \leq \bar{\alpha}$ for $i \in N_{1}$.

Theorem 5. For a minimal cover, a lifted cover inequality with $\alpha=\bar{\alpha}$ can be constructed in $O\left(n^{3}\right)$.

Proof. We have already argued that $\bar{\alpha}$ can be computed in $O(n \log n)$. Since the coefficients of the objective function of the lifting problem BLP is bounded, when computing $\alpha_{i} i \in N_{0} \cup N_{1}$, it is more efficient to solve BLP with the dual knapsack formulation $\mathrm{KP} 2$ in Sect. 3.1. After putting BLP in the form of $\mathrm{UFP}_{f}$, since $\bar{\alpha}$ is a common multiple of the coefficients $\alpha_{i} / \bar{\alpha}$ and $\bar{\alpha} \leq|C|-1<n$, from Theorem 3 the lifting problem for a single binary variable can be solved in $O\left(n^{3}\right)$ by dynamic programming. However, similar to the lifting of 0-1 knapsack cover inequalities [27], the lifting coefficients of all projected variables can also be computed in $O\left(n^{3}\right)$ by dynamic programming, since the set of variables in the knapsack problems solved for lifting are nested.

Remark 4. For the 0-1 knapsack set Zemel [27] gives an $O\left(n^{2}\right)$ algorithm to compute the lifting coefficients of a minimal cover inequality when $N_{1}=\varnothing$. However, no polynomial-time algorithm is known for constructing a lifted cover inequality for the $0-1$ knapsack set if some of the variables are projected to 1 , i.e., $N_{1} \neq \emptyset$. For the $0-1$ knapsack set $\mathrm{Gu}$ et al. [14] give an example where the lifting coefficients are bounded from below by an exponential function of $n$. In the case of the unsplittable flow arc set $\mathcal{F}_{U}$, we are able to bound the coefficients of the lifted cover inequality from above by $n$ in Lemma 4 by lifting the integer capacity variable $y$ first.

\subsubsection{Example}

Let $\mathcal{F}_{U}=\left\{(x, y) \in\{0,1\}^{5} \times \mathbb{Z}: \frac{1}{3} x_{1}+\frac{1}{3} x_{2}+\frac{1}{3} x_{3}+\frac{1}{2} x_{4}+\frac{2}{3} x_{5} \leq y\right\}$. Below we list the lifted cover inequalities of $\mathcal{F}_{U}$ that are not $c$-strong inequalities. 


\begin{tabular}{c|c|l}
$\nu$ & $\left(C, N_{0}, N_{1}\right)$ & \multicolumn{1}{|c}{ Inequalities } \\
\hline 1 & $(\{2,3,4\},\{1,5\}, \emptyset)$ & $x_{2}+x_{3}+x_{4}+x_{5} \leq 2 y$ \\
1 & $(\{1,4,5\},\{2,3\}, \emptyset)$ & $x_{1}+x_{2}+x_{4}+x_{5} \leq 2 y$ and $x_{1}+x_{3}+x_{4}+x_{5} \leq 2 y$ \\
2 & $(\{1,2,3,4\}, \emptyset,\{5\})$ & $x_{1}+x_{2}+x_{3}+x_{4}+2 x_{5} \leq 2 y+1$ \\
2 & $(\{1,2,3,5\}, \emptyset,\{4\})$ & $x_{1}+x_{2}+x_{3}+2 x_{4}+x_{5} \leq 2 y+1$
\end{tabular}

These are all of the facet-defining inequalities that can be obtained by the lifting procedure described here for minimal cover inequalities. We remark that the last two inequalities cannot be obtained by lifting any cover inequality (including non-minimal covers) unless $N_{1} \neq \emptyset$. Thus, this example illustrates that, unlike the case when $\alpha=\underline{\alpha}$, with $\alpha=\bar{\alpha}$ projecting binary variables to 1 does lead to inequalities that cannot be obtained otherwise by sequential lifting of cover inequalities. Our second remark is that all of the inequalities above except the last one are also 2-split $c$-strong inequalities. The 3 -split $c$-strong inequality $x_{1}+x_{2}+x_{3}+2 x_{4}+2 x_{5} \leq 3 y$ with $S=\{1,2,3,4,5\}$, which is facet-defining for $\operatorname{conv}\left(\mathcal{F}_{U}\right)$, however, cannot be obtained by the lifting procedure described here for any cover. Hence, this example shows that the $k$-split $c$-strong inequalities and the lifted cover inequalities are indeed different classes of inequalities.

\section{Computational results}

In order to test the effectiveness of the results in the preceding sections on network design arc sets empirically, we developed a branch-and-cut algorithm using CPLEX callable library (version 6.5.1) for solving multicommodity flow capacitated network design problems formulated as

$$
\begin{aligned}
& \min \sum_{a \in A, k \in K} g_{k a} x_{k a}+\sum_{a \in A} h_{a} y_{a} \\
& \text { s.t.: } \sum_{a \in \delta^{+}(v)} x_{k a}-\sum_{a \in \delta^{-}(v)} x_{k a}=b_{k v}, \quad \forall v \in V, \forall k \in K \text {, } \\
& \sum_{k \in K} d_{k} x_{k a} \leq c_{a} y_{a}, \quad \forall a \in A, \\
& x_{k a} \in\{0,1\}, \forall a \in A, \forall k \in K ; y_{a} \in \mathbb{Z}_{+}, \forall a \in A,
\end{aligned}
$$

where $V$ is the set of vertices, $A$ is the set of arcs, and $K$ is the set of commodities. $\delta^{+}(v)$ and $\delta^{-}(v)$ are the outbound and inbound arcs of vertex $v$, respectively, and $b_{k v}$ is 1 if $v$ is the supply vertex of commodity $k,-1$ if it is the demand vertex of $k$, and 0 otherwise. Variable $x_{k a}$ is the fraction of commodity $k$ 's demand sent through $\operatorname{arc} a$, whereas $y_{a}$ is the number of capacity units installed on arc $a$.

The branch-and-cut algorithm generates cutting planes from the splittable and unsplittable arc sets. All of the computations are done on a Sun Ultra 5 workstation with 1 hour time limit using a best-bound node selection strategy in the branch-and-bound search tree.

Our data set is based on the unsplittable multicommodity flow problem instances used in [4]. In these instances capacity is fixed and demand for commodities ranges between 5 and 60 . In order to make them capacitated network design problems, we 
introduced capacity variables with unit capacities 4, 25, 60, and 120 and unit installation costs $50,250,450$, and 720 , respectively. Here we report on our computations with 5 problems that CPLEX had the most difficulty in solving. These problem instances are available at http://ieor. berkeley. edu/ atamturk/data and their basic characteristics are summarized in Table 1. Cut-set inequalities $[18,6,1]$ are known to improve the LP relaxations of network design problems significantly; however, their separation problem is $\mathcal{N} \mathcal{P}$-hard [5]. Therefore, before solving the problems we added only the cut-set inequalities defined for one and two-node subsets of the network to the formulations and used these formulations as the basis for our comparisons.

Table 1. Problem instances

\begin{tabular}{|c|ccc|c|c|c|}
\hline \multirow{2}{*}{ Problem } & \multirow{2}{*}{ Commodities } & \multirow{2}{*}{ Nodes } & \multirow{2}{*}{ Arcs } & \multicolumn{2}{|c|}{ Variables } & \multirow{2}{*}{ Constraints } \\
\cline { 5 - 6 } & & & Flow & Capacity & \\
\hline 1 & 70 & 29 & 61 & 8540 & 61 & 2181 \\
2 & 58 & 18 & 29 & 3364 & 29 & 1120 \\
3 & 93 & 27 & 37 & 7178 & 37 & 2612 \\
4 & 87 & 24 & 42 & 7308 & 42 & 2196 \\
5 & 81 & 27 & 36 & 5832 & 36 & 2284 \\
\hline
\end{tabular}

\subsection{Experiments with splittable flow problems}

The first experiment is done to test the effectiveness of the exact separation algorithm for the residual capacity inequalities described in Sect. 2.2. Since these inequalities are developed for splittable flow network design problems, we relaxed the binary flow variables of our data set to continuous variables. Table 2 summarizes the computations with the branch-and-cut algorithm using residual capacity cuts. In this table, for each problem (prob) and capacity (cap) combination, we report the number of residual capacity cuts added (cuts), percentage improvement in the integrality gap obtained by the cuts at the root node of the search tree (gap impr), the number of nodes evaluated in the search tree (b\&b nodes), elapsed CPU time in seconds (time) or percentage gap between the best upper bound and the best lower bound at termination if the time limit is reached (endgap). The gap improvement is calculated as $100 \times \frac{\text { zroot-zprep }}{\text { zub-zprep }}$, where zprep is the objective value of the LP relaxation after preprocessing, zroot the value of the LP relaxation at the root node just before branching, and zub the value of the best integer solution known for an instance. In order to reflect the improvement obtained by the cut-set inequalities as well, we used the zprep values of formulations before adding the cut-set inequalities. In all tables columns with heading (1) show the performance of the branch-and-bound algorithm for the base formulation that includes the cut-set inequalities.

In any branch-and-cut algorithm the frequency of applying a separation routine in the search tree has an important effect on the computations. In this experiment the separation routine for the residual capacity inequalities is run only at the root node. This choice is based on our observation that most of the effective cuts are found at the root node of the search tree. Observe that a large number of cuts are added compared with the 
Table 2. Computational results with splittable flow arc sets

\begin{tabular}{|c|c|c|rr|rr|rr|}
\hline \multirow{2}{*}{ cap } & prob & \multirow{2}{*}{ cuts } & \multicolumn{2}{|c|}{ gap impr } & \multicolumn{2}{|c|}{ b\&b } & nodes & \multicolumn{2}{|c|}{ time/(endgap) } \\
& & & $(1)$ & $(2)$ & $(1)$ & $(2)$ & $(1)$ & $(2)$ \\
\hline \multirow{4}{*}{4} & 1 & 20 & 35.7 & 44.3 & 27630 & 22310 & $(0.1)$ & $(0.1)$ \\
& 2 & 17 & 25.8 & 43.2 & 6040 & 2918 & 177.4 & 85.9 \\
& 3 & 12 & 48.9 & 62.3 & 15736 & 17720 & 437.5 & 435.3 \\
& 4 & 24 & 81.8 & 81.8 & 29683 & 23282 & $(0.0)$ & $(0.0)$ \\
& 5 & 17 & 47.3 & 80.7 & 1052 & 1172 & 38.5 & 41.4 \\
\hline \multirow{6}{*}{25} & 1 & 80 & 37.7 & 56.2 & 6858 & 2929 & $(0.8)$ & $(0.8)$ \\
& 2 & 68 & 44.9 & 61.3 & 5840 & 2967 & 266.7 & 277.4 \\
& 3 & 31 & 63.1 & 75.2 & 7177 & 5476 & 206.4 & 157.5 \\
& 4 & 57 & 56.9 & 59.3 & 9554 & 7441 & $(0.7)$ & $(0.2)$ \\
& 5 & 29 & 32.3 & 68.6 & 9037 & 8384 & 285.4 & 260.4 \\
\hline \multirow{6}{*}{60} & 1 & 262 & 17.0 & 55.1 & 5403 & 967 & $(3.9)$ & $(2.7)$ \\
& 2 & 206 & 19.2 & 58.5 & 1788 & 483 & 133.6 & 161.5 \\
& 3 & 114 & 59.8 & 72.4 & 7753 & 5893 & 245.6 & 224.5 \\
& 4 & 257 & 22.4 & 55.3 & 8250 & 1832 & $(1.6)$ & $(1.4)$ \\
& 5 & 77 & 48.7 & 79.5 & 2166 & 1047 & 78.8 & 49.5 \\
\hline \multirow{6}{*}{120} & 1 & 625 & 23.2 & 52.1 & 3650 & 363 & $(17.9)$ & $(13.8)$ \\
& 2 & 500 & 26.4 & 58.1 & 39465 & 9662 & 2118 & 3411 \\
& 3 & 340 & 66.3 & 83.7 & 797 & 626 & 39.2 & 38.4 \\
& 4 & 629 & 12.9 & 47.6 & 6665 & 624 & $(10.8)$ & $(7.4)$ \\
& 5 & 209 & 55.4 & 75.9 & 1056 & 1616 & 52.4 & 78.4 \\
\hline
\end{tabular}

(1) base formulation., (2) residual cap. ineqs.

number of arcs in the problems and the number of cuts added increases with the capacity. The addition of the cuts reduces the integrality gap at the root node significantly and for almost all problems decreases the total number of nodes evaluated. For the problems that could not be solved within the time limit, endgap is smaller for all of the problems when the residual capacity cuts are added. Since the residual capacity inequalities describe the convex hull of the splittable arc sets $\mathcal{F}_{S}$ and we use an exact algorithm to separate them, the integrality gap improvement shown under heading (2) of Table 2 is the best that can be achieved by using cutting planes from individual arc sets for these instances.

\subsection{Experiments with unsplittable flow problems}

The next set of experiments are on the multicommodity unsplittable flow network design problem. First, we test the impact of the inequalities described in Sect. 3 in reducing the integrality gap at the root node of the search tree. Under headings (2), (3) and (4) of Table 3, we report the number of cuts added and the integrality gap improvement for $c$-strong inequalities, $k$-split $c$-strong inequalities, and lifted cover inequalities, respectively. As in Table 2, we use zprep values of formulations before adding the cut-set inequalities to calculate impr so that the improvement can be compared with the one obtained by cut-set inequalities, shown under heading (1) of Table 3.

Given a fractional point $(\bar{x}, \bar{y})$, in order to find violated $c$-strong inequalities, we use the fact there exists an optimal solution $S^{*}$ to the separation problem such that $i \in S^{*}$ if $\bar{x}_{i}=1$ and $i \in N \backslash S^{*}$ if $\bar{x}_{i}=0$. Therefore after fixing the variables with integral LP values, for each value of $c$ we choose the elements of $S$ in nondecreasing order of $\bar{x}_{i} a_{i}$ in a greedy fashion for the fractional variables. We observed that in the separation problem usually more than $90 \%$ of the variables are fixed by the optimality 
criteria of Proposition 2. A greedy heuristic is used to separate the knapsack cover inequalities [15] after letting $v=\lceil\bar{y}\rceil$. When lifting cover inequalities, we let the lifting coefficient of the capacity variable $\alpha=\bar{\alpha}$, since $c$-strong inequalities correspond to lifted cover inequalities with $\alpha=\alpha$. In order to find the lifting coefficients for the projected binary variables, rather than solving the lifting problems exactly, we solve their splittable relaxation as described in Sect. 2.1. To generate $k$-split $c$-strong cuts, we use the separation routine for $c$-strong inequalities, after simply multiplying the coefficients of the arc-set inequality by $k$. A preliminary test showed that the quality of $k$-split $c$-strong cuts degrade for high values of $k$. Therefore, in these computations, we set the maximum value of $k$ to 4 . Comparing headings (3) and (4) with (2) we observe that although a good number of lifted cover and $k$-split $c$-strong cuts are generated, further improvement of the integrality gap is limited. The root improvement is slightly better with $k$-split $c$-strong cuts for most of the instances. Recall that a lifted cover inequality is generated in two steps. First a violated knapsack cover inequality is found and then it is lifted with the projected variables. Even though a heuristic, the one step separation routine for the $k$-split $c$-strong inequalities may find more cuts than the knapsack cover separation that does not take into account the lifting coefficients.

Table 3. Improvement of integrality gap at the root node

\begin{tabular}{|c|c|c|c|c|c|c|c|c|}
\hline \multirow[b]{2}{*}{ cap } & \multirow[b]{2}{*}{ prob } & (1) & \multicolumn{2}{|c|}{ (2) } & \multicolumn{2}{|c|}{ (3) } & \multicolumn{2}{|c|}{ (4) } \\
\hline & & impr & cuts & impr & cuts & impr & cuts & impr \\
\hline \multirow{5}{*}{4} & 1 & 4.3 & 161 & 53.8 & 358 & 55.2 & 140 & 54.1 \\
\hline & 2 & 6.4 & 74 & 69.2 & 153 & 72.6 & 63 & 70.7 \\
\hline & 3 & 37.7 & 70 & 79.6 & 137 & 83.6 & 70 & 56.6 \\
\hline & 4 & 9.8 & 157 & 42.4 & 324 & 44.5 & 143 & 43.0 \\
\hline & 5 & 38.4 & 52 & 82.9 & 77 & 88.4 & 44 & 84.5 \\
\hline \multirow{5}{*}{25} & 1 & 0.00 & 270 & 32.2 & 633 & 34.6 & 225 & 32.7 \\
\hline & 2 & 22.8 & 119 & 57.4 & 385 & 64.6 & 106 & 62.1 \\
\hline & 3 & 54.2 & 84 & 62.0 & 218 & 63.6 & 79 & 62.0 \\
\hline & 4 & 0.00 & 204 & 12.8 & 531 & 14.4 & 165 & 13.2 \\
\hline & 5 & 17.2 & 126 & 54.1 & 218 & 56.3 & 115 & 54.1 \\
\hline \multirow{5}{*}{60} & 1 & 4.7 & 317 & 25.7 & 854 & 28.4 & 297 & 26.9 \\
\hline & 2 & 14.0 & 185 & 44.5 & 462 & 48.0 & 153 & 47.7 \\
\hline & 3 & 53.7 & 153 & 61.2 & 355 & 61.6 & 127 & 61.1 \\
\hline & 4 & 0.6 & 267 & 17.5 & 683 & 19.7 & 203 & 19.0 \\
\hline & 5 & 38.0 & 201 & 68.5 & 431 & 69.3 & 196 & 68.6 \\
\hline \multirow{5}{*}{120} & 1 & 9.4 & 486 & 30.7 & 1291 & 31.2 & 438 & 30.7 \\
\hline & 2 & 28.0 & 379 & 52.8 & 809 & 51.6 & 301 & 52.7 \\
\hline & 3 & 62.0 & 420 & 71.1 & 732 & 71.6 & 316 & 71.4 \\
\hline & 4 & 2.4 & 531 & 39.3 & 1094 & 39.7 & 437 & 38.9 \\
\hline & 5 & 35.5 & 539 & 63.0 & 746 & 63.3 & 498 & 63.0 \\
\hline
\end{tabular}

(1) base formulation, (2) $c$-strong ineqs.,

(3) $k$-split $c$-strong ineqs., (4) lifted cover ineqs.

In the next table we compare the overall performance of the branch-and-cut algorithm for $c$-strong cuts only, for $k$-split $c$-strong cuts, with $1 \leq k \leq 4$, and finally for all cuts, including the lifted covers. In Table 4 we report the number of nodes evaluated (nodes), and elapsed CPU time in seconds (time) or percentage gap between the best known upper bound and the best lower bound at termination 
if the time limit is reached (endgap). In the separation routine for $k$-split $c$-strong inequalities, for each arc we increase the value of $k$ only if no cut is found with the current value. Separation routines are run in the first 50 nodes, which correspond to nodes that are high in the search tree, because we use a best-bound node selection strategy. Comparing columns with headings (1) and (2) we see that generating $c$-strong cuts reduces the number of nodes and the overall CPU time significantly. Generating $k$-split $c$-strong inequalities and lifted cover inequalities in addition to $c$-strong inequalities generally has a positive effect. However, the additional improvement is not as significant as adding $c$-strong inequalities on the base formulation.

Table 4. Computational results with unsplittable flow arc sets

\begin{tabular}{|c|c|c|c|c|c|c|c|c|c|}
\hline cap & prob & (1) & $\begin{array}{r}\mathrm{b} \& \mathrm{~b} \\
(2)\end{array}$ & $\begin{array}{l}\text { odes } \\
\qquad(3)\end{array}$ & (4) & (1) & ime / & $\begin{array}{l}\text { endgar } \\
\text { (3) }\end{array}$ & (4) \\
\hline 4 & $\begin{array}{l}1 \\
2 \\
3 \\
4 \\
5\end{array}$ & $\begin{array}{l}18536 \\
61101 \\
78659 \\
17494 \\
99686\end{array}$ & $\begin{array}{r}4269 \\
757 \\
40359 \\
5273 \\
3345\end{array}$ & $\begin{array}{r}2521 \\
458 \\
18723 \\
7572 \\
936\end{array}$ & $\begin{array}{r}2282 \\
494 \\
16898 \\
5585 \\
1318\end{array}$ & $\begin{array}{l}(2.4) \\
(1.4) \\
(0.3) \\
(1.6) \\
(0.1)\end{array}$ & $\begin{array}{r}(1.0) \\
133.8 \\
1701 \\
(1.0) \\
160.0\end{array}$ & $\begin{array}{r}(1.0) \\
93.4 \\
819.2 \\
(1.2) \\
56.8\end{array}$ & $\begin{array}{r}(0.9) \\
117.1 \\
728.0 \\
(1.1) \\
72.1\end{array}$ \\
\hline 25 & $\begin{array}{l}1 \\
2 \\
3 \\
4 \\
5\end{array}$ & $\begin{array}{l}11283 \\
43535 \\
71023 \\
14798 \\
95088\end{array}$ & $\begin{array}{r}2084 \\
6791 \\
2399 \\
2469 \\
32464\end{array}$ & $\begin{array}{r}1351 \\
2362 \\
4877 \\
1191 \\
28679\end{array}$ & $\begin{array}{r}1268 \\
5396 \\
1896 \\
1392 \\
21012\end{array}$ & $\begin{array}{r}(12.7) \\
(8.6) \\
2687 \\
(8.8) \\
(0.1)\end{array}$ & $\begin{array}{r}(9.5) \\
2838 \\
133.4 \\
(7.9) \\
1832\end{array}$ & $\begin{array}{r}(9.3) \\
1767 \\
268.9 \\
(7.8) \\
(0.1)\end{array}$ & $\begin{array}{r}(9.3) \\
(0.0) \\
125.1 \\
(7.9) \\
1878\end{array}$ \\
\hline 60 & $\begin{array}{l}1 \\
2 \\
3 \\
4 \\
5\end{array}$ & $\begin{array}{r}9949 \\
12008 \\
9400 \\
12050 \\
7210\end{array}$ & $\begin{array}{l}4154 \\
5050 \\
9062 \\
6043 \\
2533\end{array}$ & $\begin{array}{l}2561 \\
7902 \\
6632 \\
2952 \\
4208\end{array}$ & $\begin{array}{l}1817 \\
6246 \\
6050 \\
2653 \\
1935\end{array}$ & $\begin{array}{c}(26.8) \\
842.9 \\
330.1 \\
(11.2) \\
256.8\end{array}$ & $\begin{array}{r}(25.5) \\
1397 \\
403.4 \\
(11.3) \\
134.3\end{array}$ & $\begin{array}{r}(25.0) \\
1835 \\
293.6 \\
(11.1) \\
202.4\end{array}$ & $\begin{array}{r}(24.8) \\
1890 \\
288.6 \\
(10.7) \\
106.4\end{array}$ \\
\hline 120 & $\begin{array}{l}1 \\
2 \\
3 \\
4 \\
5\end{array}$ & $\begin{array}{r}4869 \\
19082 \\
835 \\
6261 \\
22827\end{array}$ & $\begin{array}{r}968 \\
12431 \\
680 \\
1128 \\
2511\end{array}$ & $\begin{array}{r}932 \\
13208 \\
606 \\
1817 \\
2888\end{array}$ & $\begin{array}{r}720 \\
16321 \\
570 \\
1332 \\
2820\end{array}$ & $\begin{array}{r}(27.2) \\
1147 \\
42.8 \\
(14.7) \\
933.2\end{array}$ & $\begin{array}{r}(24.6) \\
2878 \\
60.0 \\
(10.0) \\
149.0\end{array}$ & $\begin{array}{r}(24.7) \\
2371 \\
53.0 \\
(8.8) \\
189.9\end{array}$ & $\begin{array}{r}(24.9) \\
(0.1) \\
67.3 \\
(9.5) \\
151.9\end{array}$ \\
\hline
\end{tabular}

(1) base formulation, (2) $c$-strong ineqs., (3) $k$-split $c$-strong ineqs., (4) all ineqs.

Based on these experimental results, our conclusion is that inequalities from the arc sets of capacitated network design problems do a reasonably good job in strengthening the LP relaxations and improving the performance of LP based search algorithms. However, inequalities that capture additional structures of the network design problems seem to be necessary for solving them more efficiently.

For the splittable flow problems, the exact separation algorithm for residual capacity inequalities empirically provides the maximum integrality gap improvements based on inequalities from arc sets. It would be interesting to know the value of the maximum possible improvement that can be obtained by using inequalities from arc sets for the unsplittable flow problems as well. One possible way of finding this bound is to solve LP relaxations of Dantzig-Wolfe reformulations of the unsplittable multicommodity flow network design problem by relaxing the demand constraints. One can solve this LP relaxation by generating columns over the pricing subproblems consisting of individual arc sets with the dynamic programming algorithm given in Sect. 3.1. 


\section{References}

1. Atamtürk, A.: On capacitated network design cut-set polyhedra. To appear in Math. Program.

2. Atamtürk, A., Rajan, D. (2000): On splittable and unsplittable flow capacitated network design arc-set polyhedra. BCOL Research Report 00.02, July 2000

3. Balas, E. (1975): Facets of the knapsack polytope. Math. Program. 8, 146-164

4. Barnhart, C., Hane, C.A., Vance, P.H. (2000): Using branch-and-price-and-cut to solve origin-destination integer multicommodity flow problems. Operations Research 48, 318-326

5. Bienstock, D. (2001): Personal communication

6. Bienstock, D., Günlük, O. (1996): Capacitated network design - Polyhedral structure and computation. INFORMS Journal on Computing 8, 243-259

7. Brockmüller, B., Günlük, O., Wolsey, L.A. (1996): Designing private line networks - Polyhedral analysis and computation. CORE Discussion Paper 9647, Université Catholique de Louvain

8. Ceria, S., Cordier, C., Marchand, H., Wolsey, L.A. (1998): Cutting planes for integer programs with general integer variables. Math. Program. 81, 201-214

9. Cordier, C., Marchand, H., Laundy, R., Wolsey, L.A. (1999): bc-opt: a branch-and-cut code for mixed integer programs. Math. Program. 86, 335-354

10. Crowder, H., Johnson, E.L., Padberg, M.W. (1983): Solving large-scale zero-one linear programming problems. Operations Research 31, 803-834

11. Garey, M.R., Johnson, D.S. (1979): Computers and Intractability: A Guide to the Theory of NPCompleteness. W.H. Freeman and Company, New York

12. Gavish, B., Altinkemer, K. (1990): Backbone network design tools with economic tradeoffs. ORSA Journal on Computing 2, 58-76

13. Grötschel, M., Lovász, L., Schrijver, A. (1981): The ellipsoid method and its consequences in combinatorial optimization. Combinatorica 1, 169-197

14. Gu, Z., Nemhauser, G.L., Savelsbergh, M.W.P. (1994): Lifted knapsack covers inequalities for 0-1 integer programs: Fast algorithms. Manuscript, Georgia Institute of Technology, Atlanta

15. Gu, Z., Nemhauser, G.L., Savelsbergh, M.W.P. (1998): Lifted cover inequalities for 0-1 integer programs: Computation. INFORMS Journal on Computing 10, 427-437

16. Hammer, P.L., Johnson, E.L., Peled, U.N. (1975): Facets of regular 0-1 polytopes. Math. Program. 8, 179-206

17. van Hoesel, S.P.M., Koster, A.M.C.A., van de Leensel, R.L.M.J., Savelsbergh, M.W.P. (2000): Polyhedral results for the edge capacity polytope. Technical Report SC 00-22, Konrad-Zuse-Zentrum für Informationstechnik Berlin

18. Magnanti, T.L., Mirchandani, P. (1993): Shortest paths, single origin-destination network design, and associated polyhedra. Networks 23, 103-121

19. Magnanti, T.L., Mirchandani, P., Vachani, R. (1993): The convex hull of two core capacitated network design problems. Math. Program. 60, 233-250

20. Marchand, H., Wolsey, L.A. (1999): The 0-1 knapsack problem with a single continuous variable. Math. Program. 85, 15-33

21. Marchand, H., Wolsey, L.A. (2001): Aggregation and mixed integer rounding to solve MIPs. Operations Research 49, 363-371

22. Nemhauser, G.L., Wolsey, L.A. (1988): Integer and Combinatorial Optimization. John Wiley and Sons, New York

23. Padberg, M.W. (1979): Covering, packing and knapsack problems. Annals of Discrete Mathematics 4, 265-287

24. Weismantel, R. (1997): On the 0/1 knapsack polytope. Math. Program. 77, 49-68

25. Wolsey, L.A. (1975): Faces for linear inequality in 0-1 variables. Math. Program. 8, 165-178

26. Wolsey, L.A. (1976): Facets and strong valid inequalities for integer programs. Operations Research 24, 367-372

27. Zemel, E. (1989): Easily computable facets of the knapsack polytope. Mathematics of Operations Research 14, 760-764 